\title{
On a family of cubic Thue equations with 5 solutions
}

\author{
by \\ ISAO WAKABAYASHI (Tokyo)
}

1. Introduction. A. Pethő $[\mathrm{P}]$ reported some results obtained by computer search for cubic Thue equations with rational integer coefficients, with positive discriminant, and representing 1 , which have many solutions. He listed a finite number of Thue equations having 9 or 6 solutions (which had been known in the literature), and two families of cubic Thue equations with at least 5 solutions. One of the two families is $x^{3}-(a+1) x^{2} y+a x y^{2}+y^{3}=1$. About this family, W. Ljunggren [L] had already pointed out that for $a \geq 2$ the pairs $(1,0),(1,1),(1,-a-1),(0,1),(a, 1)$ are its solutions. M. Mignotte and N. Tzanakis $[\mathrm{MT}]$ proved that for $a \geq 3.67 \cdot 10^{32}$ these are in fact the only solutions, and Mignotte $[\mathrm{M}]$ proved that the same holds for $a \geq 5$. The other family is $x^{3}-a^{2} x y^{2}+y^{3}=1$. About this family, we show the following.

TheOREM. For any integer $a \geq 1.35 \cdot 10^{14}$, the only integer solutions $(x, y)$ of the Thue equation

$$
x^{3}-a^{2} x y^{2}+y^{3}=1
$$

are the "trivial" solutions $(x, y)=(0,1),(1,0),\left(1, a^{2}\right),(a, 1),(-a, 1)$.

Our method for obtaining this result is based on Baker's method and consideration of a certain congruence condition. Let

$$
f(x)=x^{3}-a^{2} x+1
$$

be the corresponding inhomogeneous polynomial. Its discriminant is $4 a^{6}-27$. In the case $a \geq 2$, the discriminant is positive, and $f$ has three real zeros $\theta, \theta^{\prime}$, and $\theta^{\prime \prime}$ with

$$
\theta^{\prime \prime}<0<\theta<\theta^{\prime} .
$$

For solutions $(x, y)$ of $(1)$ such that the quotient $x / y$ is close to $\theta$, a result of [W2] (see also [W1] for a summary) obtained by the Padé approximation method is applicable, and it is easy to find all solutions with this property.

2000 Mathematics Subject Classification: Primary 11D.

Key words and phrases: cubic Thue equations. 
However, if $x / y$ is close to $\theta^{\prime}$ or $\theta^{\prime \prime}$, then it seems that the Padé approximation method is not applicable. Therefore, for these cases we use Baker's method in order to obtain an upper bound for the solutions. To obtain a lower bound for non-trivial solutions, we use a certain congruence condition. This is the main feature of our method. Then we show that if $a$ is sufficiently large, then the lower bound is greater than the upper bound, and by this we prove that for sufficiently large $a$, there is no non-trivial solution of (1) close to $\theta^{\prime}$ or $\theta^{\prime \prime}$.

2. Preliminaries. In this paper we assume that the parameter $a$ takes always positive integer values.

For $a \geq 1$ the polynomial $f(x)$ is irreducible over $\mathbb{Q}$ since $f( \pm 1) \neq 0$.

Lemma 1. For $a \geq 2$ we have

$$
\begin{aligned}
\frac{1}{a^{2}} & <\theta<\frac{1}{a^{2}}+\frac{3}{2 a^{8}}, \\
a-\frac{2}{3 a^{2}} & <\theta^{\prime}<a-\frac{1}{2 a^{2}}, \\
-a-\frac{1}{2 a^{2}} & <\theta^{\prime \prime}<-a-\frac{1}{3 a^{2}} .
\end{aligned}
$$

Proof. We obtain this by studying the sign of $f(x)$ at the given bounds.

Lemma 2. For $a \geq 1$ the only solutions of (1) with $|x| \leq 1$ or $|y| \leq 1$ are the trivial solutions.

Proof. Elementary.

Lemma 3. Let $a \geq 2$, and let $(x, y)$ be a non-trivial solution of (1). Then $x / y$ belongs to one of the intervals

$$
I=\left(\frac{1}{a^{2}}, \frac{1}{a^{2}}+\frac{5}{2 a^{8}}\right), \quad I^{\prime}=\left(a-\frac{2}{a^{2}}, a\right), \quad I^{\prime \prime}=\left(-a-\frac{1}{a^{2}},-a\right) .
$$

Proof. We easily see that $|f| \geq 1$ outside the intervals $I^{\prime}, I^{\prime \prime}$, and $\left(0,3 / a^{2}\right)$, hence, from $1=y^{3} f(x / y)$ and $|y| \geq 2, x / y$ belongs to one of these intervals. It remains to show that $x / y \notin\left(0,3 / a^{2}\right) \backslash I$. Suppose $x / y$ belongs to this interval. Then, since $|f| \geq 1 / a^{6}$ there, we have $1=\left|y^{3} f(x / y)\right| \geq\left|y^{3} / a^{6}\right|$, hence $|y| \leq a^{2}$. Further, from (1), Lemma 1 , and $|y| \geq 2$, we have $|x-\theta y|=$ $1 /\left|\left(x / y-\theta^{\prime}\right)\left(x / y-\theta^{\prime \prime}\right) y^{2}\right|<1 / a^{2}$, and from $|x| \geq 2$ and $|y| \leq a^{2}$ we have $|x-\theta y| \geq 2-\theta|y|>1-3 /\left(2 a^{6}\right)$, a contradiction.

Definition. Let $a \geq 2$. We call a solution $(x, y)$ of (1) of type $\mathrm{I} \mathrm{I}^{\prime}$, or $\mathrm{I}^{\prime \prime}$ if $x / y$ belongs to the interval $I, I^{\prime}$, or $I^{\prime \prime}$ respectively. Note that $x / y$ is close to $\theta, \theta^{\prime}$, or $\theta^{\prime \prime}$ respectively.

We see that three of the trivial solutions, except $(0,1)$ and $(1,0)$, each belong to the boundary of one of the intervals $I, I^{\prime}$, and $I^{\prime \prime}$. Our aim is to 
prove that none of these intervals contains a solution of (1) if $a$ is sufficiently large.

Lemma 4. We have $(a+\theta)(a-\theta) \theta=1$, and if $a \geq 2$, then any pair of two numbers from among $\theta, a+\theta$, and $a-\theta$ forms a system of fundamental units of the order $\mathbb{Z}[\theta]$.

Proof. See H.-J. Stender [S] for the case $a \geq 3$. For $a=2$, see for example M. Pohst and H. Zassenhaus [PZ, Table, p. 436].

3. Solution of type I. Here we shall prove that there is no solution of type I if $a \geq 2$. To show this for $a \geq 12$, we use a result of [W2] which was obtained by Padé approximation method. For type I, we can also actually use the same method as will be used later for types I' and I". However, the Padé approximation method gives a stronger result, so we use this method here.

In this section, we let $(x, y)$ be a solution of (1) of type I, that is,

$$
\frac{1}{a^{2}}<\frac{x}{y}<\frac{1}{a^{2}}+\frac{5}{2 a^{8}} .
$$

3.1. Upper bound for $y$. Since $f$ has three real zeros, and $|\theta|$ is the smallest of $|\theta|,\left|\theta^{\prime}\right|$, and $\left|\theta^{\prime \prime}\right|$, we can use Theorem 1 of [W2] for $\theta$. We recall the theorem with $a$ replaced by $-a^{2}$.

Lemma 3.1. Let $a \geq 12$. Then, for any integers $p, q(q>0)$, we have

$$
\left|\theta-\frac{p}{q}\right|>\frac{1}{2.16 \cdot 10^{5} a^{8} q^{\lambda(a)}},
$$

where

$$
\lambda(a)=1+\frac{\log (4 \sqrt{R}+12 \sqrt{6})}{\log (\sqrt{R} / 27-1 / \sqrt{R})}<3 \quad \text { with } R=4 a^{6}-27 .
$$

Further, $\lambda(a)$ is a decreasing function of $a$ and tends to 2 as $a \rightarrow \infty$.

From this lemma we easily obtain an upper bound for $|y|$, a result corresponding to Theorem 2 of [W2].

Lemma 3.2. Let $a \geq 12$. Then, for any solution $(x, y)$ of (1) of type $\mathrm{I}$, we have

$$
|y|<\left(2.161 \cdot 10^{5} a^{6}\right)^{1 /(3-\lambda(a))}
$$

with the same $\lambda(a)$ as above.

Proof. From (1), Lemma 1, and (3.1), we have

$$
|\theta-x / y|=\frac{1}{\left|\left(\theta^{\prime}-x / y\right)\left(\theta^{\prime \prime}-x / y\right) y^{3}\right|}<\frac{1}{a^{2}\left(1-1 /\left(2 a^{3}\right)\right)|y|^{3}} .
$$

Together with (3.2), this implies the lemma. 
3.2. Lower bound for $y$. From the fact that $x-\theta y$ is a unit of $\mathbb{Z}[\theta]$, we shall obtain a lower bound for $|y|$.

Lemma 3.3. Let $a \geq 2$. Then we can write

$$
x-\theta y=-(a-\theta)^{-n} \theta^{m}
$$

with positive integers $m$ and $n$, and

$$
0<\frac{3}{4} a^{3}\left(\log 3 a^{3}\right) n<m .
$$

REMARK. For the relation between $m$ and $n$, we can obtain the more precise estimate

$$
\frac{\log \left(\left(a-\theta^{\prime \prime}\right) /\left(a-\theta^{\prime}\right)\right)}{\log \left(\left|\theta^{\prime \prime}\right| / \theta^{\prime}\right)} n<m-1<\frac{\log \left(\left(a-\theta^{\prime \prime}\right) /\left(a-\theta^{\prime}\right)\right)}{\log \left(\left|\theta^{\prime \prime}\right| / \theta^{\prime}\right)} n+\frac{9}{2},
$$

and for large $a$ we can see

$$
\frac{\log \left(\left(a-\theta^{\prime \prime}\right) /\left(a-\theta^{\prime}\right)\right)}{\log \left(\left|\theta^{\prime \prime}\right| / \theta^{\prime}\right)} \sim 4 a^{3}\left(\log 4 a^{3}\right) .
$$

We omit the proof, because it is tedious, and (3.4) is sufficient for our aims.

Proof of Lemma 3.3. Since $x-\theta y$ is a unit of $\mathbb{Z}[\theta]$, by Lemma 4 it can be written as $x-\theta y= \pm(a-\theta)^{-n} \theta^{m}$ with integers $n$ and $m$. By Lemma 1 and (3.1),

$$
\frac{x-\theta^{\prime \prime} y}{x-\theta^{\prime} y}=\frac{x / y-\theta^{\prime \prime}}{x / y-\theta^{\prime}}<0
$$

hence $x-\theta y<0$ by (1). Therefore, the \pm sign in the above expression is a minus sign. We also have

$$
\frac{1-x /\left(y \theta^{\prime \prime}\right)}{1-x /\left(y \theta^{\prime}\right)}=\left(\frac{a-\theta^{\prime}}{a-\theta^{\prime \prime}}\right)^{n}\left(\frac{\theta^{\prime \prime}}{\theta^{\prime}}\right)^{m-1} .
$$

By Lemma 1 and (3.1), we can estimate the left-hand side as

$$
1<\frac{1-x /\left(y \theta^{\prime \prime}\right)}{1-x /\left(y \theta^{\prime}\right)}<1+\frac{3}{a^{3}}
$$

In fact, the last inequality can be obtained for example as follows, under the assumption $a \geq 2$ :

$$
\begin{aligned}
\frac{1-x /\left(y \theta^{\prime \prime}\right)}{1-x /\left(y \theta^{\prime}\right)} & <\frac{1+\left(\frac{1}{a^{2}}+\frac{5}{2 a^{8}}\right) /\left(a+\frac{1}{3 a^{2}}\right)}{1-\left(\frac{1}{a^{2}}+\frac{5}{2 a^{8}}\right) /\left(a-\frac{2}{3 a^{2}}\right)} \\
& <\frac{1+\frac{1}{a^{3}}\left(1+\frac{5}{2 a^{6}}\right) /\left(1+\frac{1}{3 a^{3}}\right)}{1-\frac{1}{a^{3}}\left(1+\frac{5}{2 a^{6}}\right) /\left(1-\frac{2}{3 a^{3}}\right)}<\frac{1+\frac{1}{a^{3}}}{1-\frac{1}{a^{3}}\left(1+\frac{4}{3 a^{3}}\right)}<1+\frac{3}{a^{3}} .
\end{aligned}
$$

(In the following, we also need some estimates which can be proved in the 
similar way, but the details will be omitted.) Hence we obtain

$$
1<\left(\frac{a-\theta^{\prime}}{a-\theta^{\prime \prime}}\right)^{n}\left(\frac{\theta^{\prime \prime}}{\theta^{\prime}}\right)^{m-1}<1+\frac{3}{a^{3}},
$$

and

$$
0<n \log \frac{a-\theta^{\prime}}{a-\theta^{\prime \prime}}+(m-1) \log \frac{\left|\theta^{\prime \prime}\right|}{\theta^{\prime}}<\frac{3}{a^{3}} .
$$

First we show $n \neq 0$. Suppose to the contrary that $n=0$. Then from (3.5) with $n=0$ we see that $m$ must be odd and $>1$, since $\theta^{\prime \prime}<0$ and $\left|\theta^{\prime \prime}\right| / \theta>1$ by Lemma 1. If $m=3$, then $x-\theta y=-\theta^{3}=1-a^{2} \theta$, that is, $x=1$ and $y=a^{2}$. However, this contradicts (3.1). Hence $m \geq 5$. Then from Lemma 1 we would have $\left(\theta^{\prime \prime} / \theta^{\prime}\right)^{m-1} \geq\left(\theta^{\prime \prime} / \theta^{\prime}\right)^{4}>1+3 / a^{3}$, which contradicts (3.5). Therefore, $n \neq 0$.

Next we show $n>0$. Suppose to the contrary that $n<0$. Then, since from (3.6) we have

$$
(m-1) \log \frac{\left|\theta^{\prime \prime}\right|}{\theta^{\prime}}<n \log \frac{a-\theta^{\prime \prime}}{a-\theta^{\prime}}+\frac{3}{a^{3}},
$$

and from Lemma 1 we obtain $\log \left(\left(a-\theta^{\prime \prime}\right) /\left(a-\theta^{\prime}\right)\right)>\log \left(3 a^{3}\right)$ and $\left|\theta^{\prime \prime}\right| / \theta>1$, we would have $m-1<0$, that is, $m \leq 0$. Then $|x-\theta y|=(a-\theta)^{-n} \theta^{m}>1$, which contradicts (1) by (3.1). Therefore, $n>0$.

Finally, from the first inequality of (3.6) and the estimate

$$
\frac{\log \left(\left(a-\theta^{\prime \prime}\right) /\left(a-\theta^{\prime}\right)\right)}{\log \left(\left|\theta^{\prime \prime}\right| / \theta^{\prime}\right)}>\frac{3}{4} a^{3} \log 3 a^{3},
$$

we obtain (3.4).

Lemma 3.4. Let $a \geq 2$. Then

$$
y>a^{2 a^{3} \log a} .
$$

Proof. From (3.3) we have

$$
x-\theta y=-(a-\theta)^{-n} \theta^{m} \quad \text { and } \quad x-\theta^{\prime} y=-\left(a-\theta^{\prime}\right)^{-n} \theta^{\prime m},
$$

hence

$$
\left(\theta^{\prime}-\theta\right) y=\left(a-\theta^{\prime}\right)^{-n} \theta^{\prime m}-(a-\theta)^{-n} \theta^{m},
$$

and, since $|x-\theta y|<1, n \geq 1,\left(a-\theta^{\prime}\right)^{-1}>1$, and $\theta^{\prime}-\theta>1$, using Lemma 1 and (3.4) we have

$$
\begin{aligned}
y & =\frac{\left(a-\theta^{\prime}\right)^{-n}}{\theta^{\prime}-\theta} \theta^{\prime m}-\frac{|x-\theta y|}{\theta^{\prime}-\theta}>\frac{1}{\left(a-\theta^{\prime}\right)\left(\theta^{\prime}-\theta\right)} \theta^{\prime m}-\frac{1}{\theta^{\prime}-\theta} \\
& >\frac{3 a}{2}\left(a-\frac{2}{3 a^{2}}\right)^{m}-1>\left(a-\frac{2}{3 a^{2}}\right)^{m}>\left(a-\frac{2}{3 a^{2}}\right)^{3 a^{3}\left(\log 3 a^{3}\right) / 4} \\
& >a^{2 a^{3} \log a} .
\end{aligned}
$$


3.3. Bound for a. Combining Lemmas 3.2 and 3.4 , we obtain the following.

LEMmA 3.5. If $a \geq 2$, then there is no solution of (1) of type I.

Proof. (i) Case $a \geq 12$. Suppose that there exists a solution $(x, y)$ of type I. Then from Lemmas 3.2 and 3.4, we have

$$
a^{2 a^{3} \log a}<y<\left(2.161 \cdot 10^{5} a^{6}\right)^{1 /(3-\lambda(a))},
$$

hence

$$
2 a^{3} \log a<\frac{1}{3-\lambda(a)}\left(6+\frac{\log \left(2.161 \cdot 10^{5}\right)}{\log a}\right) .
$$

However, for $a=12$ we have $\lambda(a)=2.9654$, and the above inequality does not hold. Therefore, since the right-hand side is a decreasing function of $a$, it does not hold for $a \geq 12$ either, which is a contradiction.

(ii) Case $2 \leq a \leq 11$. We used the computer software $K A N T[\mathrm{D}]$ which is based on Baker's method, and is able to solve given Thue equations, and we found that there is no solution of (1) of type I.

The lemma follows from (i) and (ii).

4. Solution of type $\mathrm{I}^{\prime}$. Here we shall prove that there is no solution of type $\mathrm{I}^{\prime}$ if $a$ is sufficiently large. In this section, we always assume $a \geq 100$, and often do not refer to this in lemmas etc. Further, we let $(x, y)$ be a solution of (1) of type $\mathrm{I}^{\prime}$, that is,

$$
a-\frac{2}{a^{2}}<\frac{x}{y}<a
$$

\subsection{Preliminaries}

Lemma 4.1. We can write

$$
x-\theta y=(a-\theta)^{n} \theta^{-m}
$$

with an even positive integer $m$ and a positive integer $n$, and $n>m+4$.

REMARK. We can obtain the more precise estimate

$$
\frac{\log \left(\left|\theta^{\prime \prime}\right| / \theta\right)}{\log \left(\left(a-\theta^{\prime \prime}\right) /(a-\theta)\right)} m-\frac{2}{a^{3}}<n-1<\frac{\log \left(\left|\theta^{\prime \prime}\right| / \theta\right)}{\log \left(\left(a-\theta^{\prime \prime}\right) /(a-\theta)\right)} m+\frac{2}{a^{3}},
$$

and for large $a$,

$$
\frac{\log \left(\left|\theta^{\prime \prime}\right| / \theta\right)}{\log \left(\left(a-\theta^{\prime \prime}\right) /(a-\theta)\right)} \sim \frac{3 \log a}{\log 2} .
$$

We omit the proof. 
Proof of Lemma 4.1. Since $x-\theta y$ is a unit of $\mathbb{Z}[\theta]$, by Lemma 4 it can be written as $x-\theta y= \pm(a-\theta)^{n} \theta^{-m}$ with integers $n$ and $m$. Hence

$$
\frac{x / y-\theta^{\prime \prime}}{x / y-\theta}=\frac{x-\theta^{\prime \prime} y}{x-\theta y}=\left(\frac{a-\theta^{\prime \prime}}{a-\theta}\right)^{n}\left(\frac{\theta^{\prime \prime}}{\theta}\right)^{-m} .
$$

By Lemma 1 and (4.1), the left-hand side is positive. This implies that $m$ is even, since only $\theta^{\prime \prime}$ is negative on the right-hand side. Also we have $\left(x-\theta^{\prime \prime} y\right)(x-\theta y)>0$, hence $x-\theta^{\prime} y>0$ by (1). Therefore, the \pm sign in the above expression is a plus sign.

By Lemma 1 and (4.1), we have $3 / 2<\left(x / y-\theta^{\prime \prime}\right) /(x / y-\theta)<9 / 4$. Hence

$$
\log \frac{3}{2}<n \log \frac{a-\theta^{\prime \prime}}{a-\theta}-m \log \frac{\left|\theta^{\prime \prime}\right|}{\theta}<\log \frac{9}{4} .
$$

We first prove that $m>0$. If $m=0$, then, since $3 / 2<\left(a-\theta^{\prime \prime}\right) /(a-\theta)<$ $9 / 4$ by Lemma 1, we would have $n=1$ from (4.3). Hence $x-\theta y=a-\theta$, and we would have $x=a, y=1$, which contradicts (4.1). If $m<0$, then, since $\left|\theta^{\prime \prime}\right| / \theta>a^{3}>9 / 4$ by Lemma 1 , we would have $n<0$ by (4.3). Hence $x-\theta^{\prime} y=\left(a-\theta^{\prime}\right)^{n} \theta^{\prime-m}>1$. On the other hand, $\left|x-\theta^{\prime} y\right|<1$ by (1) and (4.1), a contradiction. Therefore, $m>0$.

Next, from Lemma 1, the first inequality of (4.3), and the inequality $m \geq 2$, we obtain

$$
n-m-4>m\left(\frac{\log \left(\left|\theta^{\prime \prime}\right| / \theta\right)}{\log \left(\left(a-\theta^{\prime \prime}\right) /(a-\theta)\right)}-1\right)-4>0,
$$

which completes the proof of the lemma.

4.2. Linear form and lower bound. From Siegel's identity

$$
\left(\theta^{\prime}-\theta^{\prime \prime}\right)(x-\theta y)+\left(\theta^{\prime \prime}-\theta\right)\left(x-\theta^{\prime} y\right)+\left(\theta-\theta^{\prime}\right)\left(x-\theta^{\prime \prime} y\right)=0
$$

and (4.2), we obtain

$$
\begin{aligned}
\Lambda & :=\log \frac{\theta^{\prime}-\theta^{\prime \prime}}{\theta^{\prime}-\theta}+n \log \frac{a-\theta}{a-\theta^{\prime \prime}}+m \log \left|\theta^{\prime \prime} / \theta\right| \\
& =\log \left(1+\frac{\left(\theta-\theta^{\prime \prime}\right)\left(x-\theta^{\prime} y\right)}{\left(\theta^{\prime}-\theta\right)\left(x-\theta^{\prime \prime} y\right)}\right) .
\end{aligned}
$$

For an algebraic number $\alpha$, we denote by $h(\alpha)$ the absolute logarithmic height.

We recall the following result of M. Waldschmidt [Wal, Cor. 1.5, p. 212].

Lemma 4.2. Let $\Lambda$ be a linear form in the principal values of logarithm of $l$ positive algebraic numbers $\alpha_{1}, \ldots, \alpha_{l}$, with rational integer coefficients $b_{1}, \ldots, b_{l}\left(b_{l} \neq 0\right)$. Put $D=\left[\mathbb{Q}\left(\alpha_{1}, \ldots, \alpha_{l}\right): \mathbb{Q}\right]$. Let $A_{1}, \ldots, A_{l}, A, E, \varrho$ be positive numbers satisfying

$$
\log A_{i} \geq h\left(\alpha_{i}\right) \quad(1 \leq i \leq l), \quad A=\max \left\{A_{1}, \ldots, A_{l}\right\},
$$


and

$$
e \leq E \leq \min \left\{A_{1}^{D}, \ldots, A_{l}^{D}, \frac{l D}{\varrho}\left(\sum_{i=1}^{l} \frac{\left|\log \alpha_{i}\right|}{\log A_{i}}\right)^{-1}\right\}
$$

Put

$$
\begin{gathered}
M=\max _{1 \leq i<l}\left\{\frac{\left|b_{l}\right|}{\log A_{i}}+\frac{\left|b_{i}\right|}{\log A_{l}}\right\}, \\
Z_{0}=\max \{7+3 \log l,(\log E) / D, \log (D / \log E)\}, \\
G_{0}=\max \left\{4 l Z_{0}, \log M\right\}, \\
U_{0}=\max \left\{D^{2} \log A, D^{l+2} G_{0} Z_{0} \log A_{1} \cdots \log A_{l}(\log E)^{-l-1}\right\} .
\end{gathered}
$$

Then

$$
\log |\Lambda| \geq-1500 \cdot 2^{2 l} l^{3 l+5}(1+1 / \varrho)^{l} U_{0} .
$$

Further, in the case $l=3$, the number 1500 can be replaced by 1320 .

LEMMA 4.3. A lower bound for the linear form (4.4) is given by

$$
\begin{aligned}
\log |\Lambda|> & -4.91 \cdot 10^{16}\left(1+\frac{\log 2}{3 \log a}\right)\left(1+\frac{2 \log 2}{3 \log a}\right)\left(1+\frac{1}{6 a^{3} \log a}\right) \\
& \times(\log a)^{3} \max \{12(7+3 \log 3), \log n\} .
\end{aligned}
$$

Proof. We put

$$
\alpha_{1}=\frac{\theta^{\prime}-\theta^{\prime \prime}}{\theta^{\prime}-\theta}, \quad \alpha_{2}=\frac{a-\theta}{a-\theta^{\prime \prime}}, \quad \alpha_{3}=\left|\theta^{\prime \prime}\right| / \theta
$$

and apply Lemma 4.2. We have $l=3, D=6, b_{1}=1, b_{2}=n, b_{3}=m$. Using Lemma 1, we have the following estimates, and we define $A_{i}$ as indicated:

$$
\begin{aligned}
h\left(\alpha_{1}\right) & \leq 2 h\left(\theta^{\prime}-\theta\right)=\frac{2}{3} \log \left(\left(\theta^{\prime}-\theta\right)\left(\theta^{\prime}-\theta^{\prime \prime}\right)\left(\theta-\theta^{\prime \prime}\right)\right) \\
& <2 \log a+\frac{2}{3} \log 2=\log A_{1}, \\
h\left(\alpha_{2}\right) & =\frac{1}{3} \log \left(\theta^{\prime}\left(a+\theta^{\prime}\right)\left(a-\theta^{\prime \prime}\right)\right)<\log a+\frac{2}{3} \log 2=\log A_{2}, \\
h\left(\alpha_{3}\right) & =\frac{1}{3} \log \frac{\left|\theta^{\prime \prime}\right|}{\theta}<\log a+\frac{1}{6 a^{3}}=\log A_{3} .
\end{aligned}
$$

Using Lemma 1 (always under the assumption $a \geq 100$ ) we also have

$$
\sum_{i=1}^{3} \frac{\left|\log \alpha_{i}\right|}{\log A_{i}}<3.21
$$

We put $\varrho=0.63, E=8.9$. Then $Z_{0}=7+3 \log 3$. Using the inequality $m<n-4$ of Lemma 4.1, we also have $M<n$. Hence

$$
G_{0} \leq \max \{12(7+3 \log 3), \log n\} .
$$


We also have

$$
U_{0}=D^{5} G_{0} Z_{0} \log A_{1} \log A_{2} \log A_{3}(\log E)^{-4} .
$$

These data and Lemma 4.2 imply Lemma 4.3.

4.3. Upper bound for $\Lambda$

LEMMA 4.4. We have

$$
\log |\Lambda|<-3 n \log a .
$$

Proof. We estimate the right-hand side of (4.4). By Lemmas 1 and 4.1,

$$
\begin{aligned}
0 & <\frac{\left(\theta-\theta^{\prime \prime}\right)\left(x-\theta^{\prime} y\right)}{\left(\theta^{\prime}-\theta\right)\left(x-\theta^{\prime \prime} y\right)}=\frac{\theta-\theta^{\prime \prime}}{\theta^{\prime}-\theta} \cdot \frac{\left(a-\theta^{\prime}\right)^{n} \theta^{\prime \prime m}}{\left(a-\theta^{\prime \prime}\right)^{n} \theta^{\prime m}} \\
& <\frac{\theta-\theta^{\prime \prime}}{\theta^{\prime}-\theta} \cdot\left(\frac{a-\theta^{\prime}}{a-\theta^{\prime \prime}}\right)^{n}\left(\frac{\left|\theta^{\prime \prime}\right|}{\theta^{\prime}}\right)^{n-4}<\frac{1}{a^{3 n}},
\end{aligned}
$$

which implies the lemma.

4.4. Lower bound for $n$. By considering some congruence condition, we shall obtain a lower bound for $n$. (This means a lower bound for $|y|$ of solutions $(x, y)$ of type $\mathrm{I}^{\prime}$.) This is our main point in the proof of the Theorem.

LEMMA 4.5. We have $n>\sqrt{2 a^{3}}$.

Proof. Since $n>m+4$ by Lemma 4.1 , we put $n-m-2=3 k+i$ with $i=0,1$, or 2 , and $k>0$. We write $(a-\theta)^{n} \theta^{-m}$ as a linear combination of $1, \theta$, and $\theta^{2}$, and calculate the coefficient of $\theta^{2}$ in the $\operatorname{ring}\left(\mathbb{Z} / a^{3+i} \mathbb{Z}\right)[\theta]$. Note that there is no relation between $1, \theta$, and $\theta^{2}$ in this ring, and that $\theta^{-1}$ belongs to this ring. We have

$$
(a-\theta)^{n}=(-\theta)^{n}+n a(-\theta)^{n-1}+\left(\begin{array}{l}
n \\
2
\end{array}\right) a^{2}(-\theta)^{n-2}+\ldots,
$$

and

$$
\begin{aligned}
x-\theta y & =(a-\theta)^{n} \theta^{-m} \\
& =(-1)^{n}\left(\theta^{2}-n a \theta+\left(\begin{array}{l}
n \\
2
\end{array}\right) a^{2}-\left(\begin{array}{l}
n \\
3
\end{array}\right) a^{3} \theta^{-1}+\left(\begin{array}{l}
n \\
4
\end{array}\right) a^{4} \theta^{-2}-\ldots\right) \theta^{n-m-2} .
\end{aligned}
$$

Since

$$
\begin{aligned}
\theta^{n-m-2} & =\theta^{3 k+i}=\left(-1+a^{2} \theta\right)^{k} \theta^{i} \\
& =(-1)^{k}\left(1-k a^{2} \theta+\left(\begin{array}{l}
k \\
2
\end{array}\right) a^{4} \theta^{2}-\ldots\right) \theta^{i}
\end{aligned}
$$


we see that the coefficient of $\theta^{2}$ of $(a-\theta)^{n} \theta^{-m}$ is

$$
\equiv \begin{cases}(-1)^{n+k} \bmod a^{3} & \text { for } i=0, \\
(-1)^{n+k+1} n a \bmod a^{4} & \text { for } i=1, \\
(-1)^{n+k}\left(1+k+\left(\begin{array}{c}
n \\
2
\end{array}\right)\right) a^{2} \bmod a^{5} & \text { for } i=2,\end{cases}
$$

which should be zero. However, if $n \leq \sqrt{2 a^{3}}$, then it would not be zero for all cases, since for example, for $i=2$ we have

$$
0<1+k+\left(\begin{array}{l}
n \\
2
\end{array}\right)=1+(n-m-4) / 3+\left(\begin{array}{l}
n \\
2
\end{array}\right)<n^{2} / 2 \leq a^{3} .
$$

This yields the lemma.

4.5. Bound for a. Combining Lemmas 4.3-4.5, we obtain

LEMMA 4.6. If $a \geq 1.34 \cdot 10^{14}$, then there is no solution of (1) of type $\mathrm{I}^{\prime}$.

Proof. Let $a \geq 1.34 \cdot 10^{14}$. Suppose that there exists a solution $(x, y)$ of type I'. Then, through the linear form (4.4), we have, from Lemmas 4.3 and 4.4 ,

$$
\begin{gathered}
3 n \log a<4.91 \cdot 10^{16}\left(1+\frac{\log 2}{3 \log a}\right)\left(1+\frac{2 \log 2}{3 \log a}\right)\left(1+\frac{1}{6 a^{3} \log a}\right) \\
\times(\log a)^{3} \max \{12(7+3 \log 3), \log n\} .
\end{gathered}
$$

We divide the proof into two cases.

(i) Case $12(7+3 \log 3) \geq \log n$. By Lemma 4.5 and the above inequality,

$$
\begin{aligned}
\sqrt{2 a^{3}}<n< & 4.91 \cdot 4(7+3 \log 3) \cdot 10^{16}\left(1+\frac{\log 2}{3 \log a}\right) \\
& \times\left(1+\frac{2 \log 2}{3 \log a}\right)\left(1+\frac{1}{6 a^{3} \log a}\right)(\log a)^{2} .
\end{aligned}
$$

However, this does not hold for $a \geq 1.34 \cdot 10^{14}$.

(ii) Case $12(7+3 \log 3)<\log n$. We have

$$
n<1.64 \cdot 10^{16}\left(1+\frac{\log 2}{3 \log a}\right)\left(1+\frac{2 \log 2}{3 \log a}\right)\left(1+\frac{1}{6 a^{3} \log a}\right)(\log a)^{2} \log n .
$$

Hence, by Lemma 4.5 we obtain

$$
\begin{aligned}
\frac{\sqrt{2 a^{3}}}{\log \sqrt{2 a^{3}}} & <\frac{n}{\log n} \\
& <1.64 \cdot 10^{16}\left(1+\frac{\log 2}{3 \log a}\right)\left(1+\frac{2 \log 2}{3 \log a}\right)\left(1+\frac{1}{6 a^{3} \log a}\right)(\log a)^{2} .
\end{aligned}
$$

However, this does not hold for $a \geq 6.96 \cdot 10^{13}$, which concludes the proof. 
5. Solution of type $I^{\prime \prime}$. Here we shall prove that there is no solution of type $\mathrm{I}^{\prime \prime}$ if $a$ is sufficiently large. The method is exactly the same as for type $\mathrm{I}^{\prime}$, so we shall give only a brief sketch. In this section, we always assume $a \geq 100$. Further, we let $(x, y)$ be a solution of (1) of type $\mathrm{I}^{\prime \prime}$, that is,

$$
-a-\frac{1}{a^{2}}<\frac{x}{y}<-a \text {. }
$$

\subsection{Preliminaries}

LEMMA 5.1. We can write

$$
x-\theta y= \pm(a+\theta)^{n} \theta^{-m}
$$

with positive integers $m$ and $n$, and $n>m+4$.

REMARK. We can obtain the more precise estimate

$$
\frac{\log \left(\theta^{\prime} / \theta\right)}{\log \left(\left(a+\theta^{\prime}\right) /(a+\theta)\right)} m-\frac{2}{a^{3}}<n-1<\frac{\log \left(\theta^{\prime} / \theta\right)}{\log \left(\left(a+\theta^{\prime}\right) /(a+\theta)\right)} m+\frac{1}{a^{3}},
$$

and for large $a$,

$$
\frac{\log \left(\theta^{\prime} / \theta\right)}{\log \left(\left(a+\theta^{\prime}\right) /(a+\theta)\right)} \sim \frac{3 \log a}{\log 2} .
$$

We omit the proof.

Proof of Lemma 5.1. By Lemma 4 we can write $x-\theta y= \pm(a+\theta)^{n} \theta^{-m}$ with integers $n$ and $m$. Hence

$$
\frac{\theta^{\prime}-x / y}{\theta-x / y}=\left(\frac{a+\theta^{\prime}}{a+\theta}\right)^{n}\left(\frac{\theta^{\prime}}{\theta}\right)^{-m} .
$$

By Lemma 1 and (5.1), the left-hand side lies in (3/2, 9/4), hence

$$
\log \frac{3}{2}<n \log \frac{a+\theta^{\prime}}{a+\theta}-m \log \frac{\theta^{\prime}}{\theta}<\log \frac{9}{4} .
$$

This implies $m>0$. In fact, if $m=0$, then since $3 / 2<\left(a+\theta^{\prime}\right) /(a+\theta)<9 / 4$ by Lemma 1, we would have $n=1$ from (5.3), hence $x= \pm a, y=\mp 1$, so $x / y=-a$, which contradicts (5.1). If $m<0$, then since $\theta^{\prime} / \theta>9 / 4$, we would have $n<0$. Hence $\left|x-\theta^{\prime \prime} y\right|=\left|\left(a+\theta^{\prime \prime}\right)^{n} \theta^{\prime \prime}-m\right|>1$, which contradicts the inequality $\left|x-\theta^{\prime \prime} y\right|<1$ implied by (1) and (5.1). Therefore, $m>0$.

As before, we obtain $n-m-4>0$ by Lemma 1 , the first inequality of (5.3), and the inequality $m \geq 1$.

5.2. Linear form and lower bound. From Siegel's identity and (5.2), we obtain

$$
\begin{aligned}
\Lambda & :=\log \frac{\theta^{\prime}-\theta^{\prime \prime}}{\theta-\theta^{\prime \prime}}+n \log \frac{a+\theta}{a+\theta^{\prime}}+m \log \left(\theta^{\prime} / \theta\right) \\
& =\log \left(1+\frac{\left(\theta-\theta^{\prime}\right)\left(x-\theta^{\prime \prime} y\right)}{\left(\theta^{\prime \prime}-\theta\right)\left(x-\theta^{\prime} y\right)}\right) .
\end{aligned}
$$


LEMma 5.2. A lower bound for the linear form (5.4) is given by

$$
\begin{aligned}
\log |\Lambda|> & -4.91 \cdot 10^{16}\left(1+\frac{\log 2}{3 \log a}\right)\left(1+\frac{\log 6}{3 \log a}\right)\left(1+\frac{1}{6 a^{3} \log a}\right) \\
& \times(\log a)^{3} \max \{12(7+3 \log 3), \log n\} .
\end{aligned}
$$

Proof. We put

$$
\alpha_{1}=\frac{\theta^{\prime}-\theta^{\prime \prime}}{\theta-\theta^{\prime \prime}}, \quad \alpha_{2}=\frac{a+\theta}{a+\theta^{\prime}}, \quad \alpha_{3}=\theta^{\prime} / \theta,
$$

and apply Lemma 4.2. We have $l=3, D=6, b_{1}=1, b_{2}=n, b_{3}=m$. Using Lemma 1 , we have the following estimates, and we define $A_{i}$ as indicated:

$$
\begin{aligned}
& h\left(\alpha_{1}\right) \leq 2 h\left(\theta^{\prime}-\theta\right)<2 \log a+\frac{2}{3} \log 2=\log A_{1}, \\
& h\left(\alpha_{2}\right)=\frac{1}{3} \log \left(\left(a+\theta^{\prime}\right) /\left|a+\theta^{\prime \prime}\right|\right)<\log a+\frac{1}{3} \log 6=\log A_{2}, \\
& h\left(\alpha_{3}\right)=h\left(\theta^{\prime \prime} / \theta\right)<\log a+\frac{1}{6 a^{3}}=\log A_{3} .
\end{aligned}
$$

Using Lemma 1, we also have

$$
\sum_{i=1}^{3} \frac{\left|\log \alpha_{i}\right|}{\log A_{i}}<3.21 .
$$

We put $\varrho=0.63, E=8$.9. Then $Z_{0}=7+3 \log 3$.

Using the inequality $m<n-4$, we also have $M<n$. Hence

$$
G_{0} \leq \max \{12(7+3 \log 3), \log n\} .
$$

We also have

$$
U_{0}=D^{5} G_{0} Z_{0} \log A_{1} \log A_{2} \log A_{3}(\log E)^{-4} .
$$

These data and Lemma 4.2 imply Lemma 5.2.

5.3. Upper bound for $\Lambda$

Lemma 5.3. We have

$$
\log |\Lambda|<-3 n \log a .
$$

Proof. We estimate the right-hand side of (5.4) by Lemmas 1 and 5.1.

5.4. Lower bound for $n$. By considering the same congruence condition as before, we obtain a lower bound for $n$.

LEMMA 5.4. We have $n>\sqrt{2 a^{3}}$.

Proof. Since $n>m+4$ by Lemma 5.1 , we put $n-m-2=3 k+i$ with $i=0,1$, or 2 , and $k>0$. We write $(a+\theta)^{n} \theta^{-m}$ as a linear combination of 
$1, \theta$, and $\theta^{2}$, and calculate the coefficient of $\theta^{2}$ in the $\operatorname{ring}\left(\mathbb{Z} / a^{3+i} \mathbb{Z}\right)[\theta]$. We have

$$
\begin{aligned}
\pm(x-\theta y) & =(a+\theta)^{n} \theta^{-m} \\
& =\left(\theta^{2}+n a \theta+\left(\begin{array}{l}
n \\
2
\end{array}\right) a^{2}+\left(\begin{array}{l}
n \\
3
\end{array}\right) a^{3} \theta^{-1}+\left(\begin{array}{l}
n \\
4
\end{array}\right) a^{4} \theta^{-2}+\ldots\right) \theta^{n-m-2},
\end{aligned}
$$

and by (4.5) the coefficient of $\theta^{2}$ of $(a+\theta)^{n} \theta^{-m}$ is

$$
\equiv \begin{cases}(-1)^{k} \bmod a^{3} & \text { for } i=0, \\
(-1)^{k} n a \bmod a^{4} & \text { for } i=1, \\
(-1)^{k}\left(1+k+\left(\begin{array}{c}
n \\
2
\end{array}\right)\right) a^{2} \bmod a^{5} & \text { for } i=2,\end{cases}
$$

which should be zero. However, if $n \leq \sqrt{2 a^{3}}$, then it is not zero for any case, which yields the lemma.

5.5. Bound for a. Combining Lemmas 5.2, 5.3, and 5.4, we obtain the following.

Lemma 5.5. If $a \geq 1.35 \cdot 10^{14}$, then there is no solution of (1) of type $\mathrm{I}^{\prime \prime}$.

Proof. Let $a \geq 1.35 \cdot 10^{14}$. Suppose that there exists a solution $(x, y)$ of type $\mathrm{I}^{\prime \prime}$. Then, from Lemmas 5.2 and 5.3, we obtain

$$
\begin{aligned}
3 n \log a< & 4.91 \cdot 10^{16}\left(1+\frac{\log 2}{3 \log a}\right)\left(1+\frac{\log 6}{3 \log a}\right)\left(1+\frac{1}{6 a^{3} \log a}\right) \\
& \times(\log a)^{3} \max \{12(7+3 \log 3), \log n\} .
\end{aligned}
$$

By Lemma 5.4 and this inequality, the lemma is proven in exactly the same way as Lemma 4.6.

Conclusion. Lemmas 3, 3.5, 4.6, and 5.5 yield the Theorem.

\section{References}

[D] M. Daberkow, C. Fieker, J. Klüners, M. Pohst, K. Roegner and K. Wildanger, KANT V4, J. Symbolic Comput. 24 (1997), 267-283.

[L] W. Ljunggren, Einige Bemerkungen über die Darstellung ganzer Zahlen durch binäre kubische Formen mit positiver Diskriminante, Acta Math. 75 (1943), 1-21.

[M] M. Mignotte, Pethö's cubics, Publ. Math. Debrecen 56 (2000), 481-505.

[MT] M. Mignotte and N. Tzanakis, On a family of cubics, J. Number Theory 39 (1991), 41-49.

[P] A. Pethő, On the representation of 1 by binary cubic forms with positive discriminant, in: Number Theory (Ulm, 1987), H. P. Schlickewei and E. Wirsing (eds.), Lecture Notes in Math. 1380, Springer, 1989, 185-196.

[PZ] M. Pohst and H. Zassenhaus, Algorithmic Algebraic Number Theory, Cambridge Univ. Press, Cambridge, 1989.

[S] H.-J. Stender, Einheiten für eine allgemeine Klasse total reeller algebraischer Zahlkörper, J. Reine Angew. Math. 257 (1972), 151-178. 
[W1] I. Wakabayashi, On families of cubic Thue equations, in: Analytic Number Theory, C. Jia and K. Matsumoto (eds.), Developments Math. 6, Kluwer, Dordrecht, 2002, 359-377.

[W2] -, Cubic Thue inequalities with negative discriminant, J. Number Theory 97 (2002), 222-251.

[Wal] M. Waldschmidt, Minorations de combinaisons linéaires de logarithmes de nombres algébriques, Canad. J. Math. 45 (1993), 176-224.

Faculty of Engineering

Seikei University

Kichijoji Kitamachi, Musashino-shi

Tokyo 180-8633, Japan

E-mail: wakaba@ge.seikei.ac.jp 